\title{
Simulation of casting deformation based on mold surface element method
}

\author{
Tao Chen, Yu-long Tang, *Dun-ming Liao, Liu Cao, Fei Sun, Zi-hao Teng, and Di Wu \\ State Key Laboratory of Material Processing and Die \& Mold Technology, Huazhong University of Science and Technology, China
}

\begin{abstract}
Deformation of casting during the solidification process has puzzled many engineers and scientists for years. In order to attain the goal of near-net forming by casting, numerical simulation is a powerful tool. Traditional methods compute the thermal stress of both the casting and the mold. This method suffers the problem of massive calculation and failure of convergence. This paper proposes an improved Mold Surface Element Method, the main idea of which is to use the surface elements instead of body elements to express the interactions between the casting and the mold. The proposed method shows a high computation efficiency and provides satisfactory precision for engineering. Two practical casting products were used to verify the proposed method. The simulated results agree well with those observed in practical products. The proposed method is believed to benefit production practice and to provide theoretical guidance.
\end{abstract}

Key words: mold surface element method; thermal stress simulation of casting; casting deformation
CLC numbers: TP391.9
Document code: A
Article ID: 1672-6421(2017)01-028-06

$\mathrm{N}$ ear net shape (NNS) manufacturing has shown promise for the future of foundry industry, decreasing machining allowance and increasing the efficiency of production dramatically. The impact of thermal stress during casting solidification on the defects and deformation is of great concern. Engineers and researchers make great efforts to try to precisely control the deformation of castings ${ }^{[1]}$, but the deformation of castings is still a difficult puzzle. Numerical simulation of thermal stress in casting is an effective approach to predict the distribution of thermal stress and the deformation of castings, assessing the soundness of products ${ }^{[2]}$. Based on the results of numerical simulation, the process designer can modify the shape of the original castings so as to compensate for the deformation during solidification while preserving the soundness of the castings ${ }^{[3]}$.

Many studies have been made on the deformation resulting from thermal stress. Wang Yueping ${ }^{[4]}$ used ProCAST software to simulate the casting process of turbine blades made of Ni-based superalloy. He compared the results of numerical simulation and the measurements taken in the experiments, providing

\section{*Dun-ming Liao}

Male, born in 1973, professor, Ph.D. His research interests mainly focus on casting process simulation, especially on casting stress simulation and casting process CAD.

E-mail: liaodunming@hust.edu.cn

Received: 2016-05-09; Accepted: 2016-10-08 suggestions for process optimization so as to reduce the residual stress and improve the geometrical precision and stability. By applying ANSYS to the thermal stress simulation, Cheng Jianguo, et al. ${ }^{[5]}$ obtained the distribution of displacement differences in the casting. They changed the geometry of the casting according to the reference points and achieved the desired shape with deformation supplements. Li Hui, et al. ${ }^{[6]}$ used ProCAST to simulate the casting process, analyzing the influences of pouring temperature and mold-shaking temperature on the residual stress and deformation of castings. Wang Peng ${ }^{[7]}$ studied the parts of large thin-walled cylindrical shells of ZL205A alloy. The deformation during the solidification of the casting and quenching after the solidification were simulated by commercial simulation software ProCAST and heat treatment modules in SYSWELD simulation software, and rules in the deformation were achieved. Cui Xinpeng et al. ${ }^{[8]}$ simulated the temperature field, stress field and casting deformation of Ti-Alloy framework castings during the pouring and solidification processes based on ProCAST software. Their simulation results revealed that the temperature of the upper and inner part of the framework castings was much lower than that of the bottom part and the pouring gates. Wei Donghai et al. ${ }^{[9]}$ used the simulation software JSCAST to analyze the stress and strain of stress-box specimens made of gray iron (HT250, HT300, HT350) and ductile iron (QT400-18, QT600-3, QT800-2). They concluded from the simulation results that the larger elastic modulus led 
to the larger residual stress in the castings.

In the aforementioned studies, the boundary conditions of the mold used a simplified model, either rigid or with no resistance, leading to results of relatively low precision. For some cases with complicated casting geometries and real mold conditions, the calculations of the thermal stress may become difficult tasks. Even the sophisticated commercial software PROCAST may suffer from the problems of convergence failure. Therefore, we need new approaches to guarantee high accuracy and calculation convergence for arbitrary complex casting processes.

During the solidification process of castings, the casting and the mold (or core) interact with each other in complex ways ${ }^{[10]}$. The casting may separate from the mold or merge into the mold, and there are frictions on the interfaces between them. Modeling of the interaction between casting and mold is critical for the precision of numerical simulation. There are three approaches of modeling:

(a) The mold is considered as rigid or having no resistance.

This kind of approach is easy to implement $\mathbf{b}$ with a larg amont of error ${ }^{[1,12]}$. A rigid mold is fit for the case of a metal mold; while the mold with no resistance is fit for the case of a soft sand mold.

(b) The effect of the mold is simplified with a well-designed mathematical model.

This kind of approach is simple but only applies in some particular cases, i.e. some cases with special casting geometries ${ }^{[13,14]}$.

(c) The mold is modeled as contact elements.

This kind of approach has the highest numerical precision as it accords to the real interactions between casting and mold. However, this method involves complicated contact problems with nonlinearity; therefore the calculation may fail to converge for some complicated casting processes ${ }^{[15-17]}$.

We propose a new kind of approach called mold surface element (MSE) method, which deems the mold as boundary elements around the casting (Fig. 1). The proposed method is capable of simulating complicated contact problems between the casting and mold (or core) with desirable precision and computational efficiency.

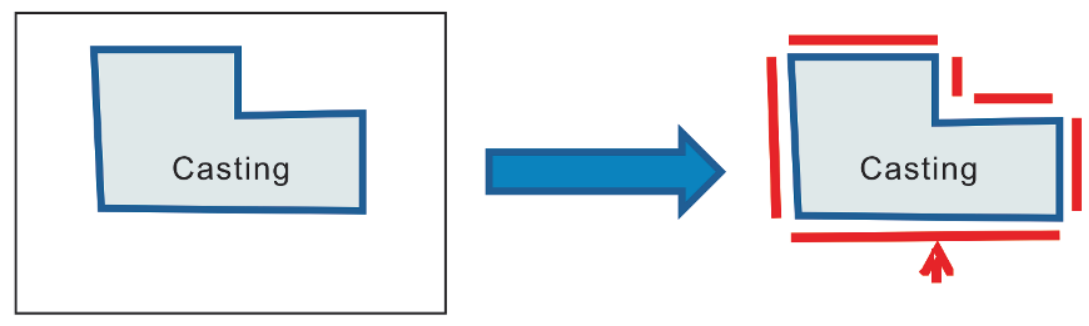

Fig. 1: Schematic of Mold Surface Element Method

\section{Mold surface element method}

The main id a of the mold sn face element (MSE) method is to use the surface elements instead of body elements to express the interactions between the casting and the mold. The surface elements are picked up from the body elements in the mesh of the casting. Then the mechanical boundary conditions can be set on these surface elements according to the casting process. In the common method of "contact elements", the thermal stress calculation involves not only the casting but also the mold and the contact problems at the interfaces. Thus the present method d mand fewer calch ations than the contact element method improving the calculation efficiency and stability.

The MSE method features several advantages:

(1) Compared with the method of a rigid mold or a mold with no resistance, the MSE method can describe the complex boundary condition of the mold efficiently and effectively, such as the casting process with mold parts of several kinds of materials.

(2) Compared with the contact element method, there is no calculation for the strain and the stress of the mold in the present method, thus the total computation can be reduced and the efficiency and convergency can be improved.

(3) All the restrictions of displacement are set automatically, assuring the non-singularity of the stiffness matrix.

The following section discusses how to integrate the mold surface element method into the traditional thermal stress equations.

The thermal stress and deformation evolution in casting is controlled by the following three equations

(1) Constitution equation

$$
\sigma=D^{e p}\left(\varepsilon-\varepsilon_{0}\right)
$$

where, $\sigma$ is the tensor of stress, $D^{e p}$ is the tensor of elastoplastic constitution, $\varepsilon$ is the tensor of strain, and $\varepsilon_{0}$ is the tensor of initial strain caused by temperature variation.

(2) Geometry equation

$$
\varepsilon=\mathbf{L u}
$$

where, $\mathbf{L}$ is the tensor of geometric relation, $\mathbf{u}$ is the displacement.

(3) Energy equation

$$
\Pi_{p}=\frac{1}{2} \int_{\Omega} \sigma\left(\varepsilon-\varepsilon_{0}\right) d \Omega-\int_{\Omega} \mathbf{u} \cdot \mathbf{F} d \Omega-\int_{\Gamma} \mathbf{u} \cdot \mathbf{T} d \Gamma
$$

where, $\Pi_{p}$ is the total potential energy, $\Omega$ is the region of solution, $\mathbf{F}$ is the body force, and $\mathbf{T}$ is the surface force.

In the finite element method, the displacement $\mathbf{u}$ can be discretized as Eq. (4).

$$
\mathbf{u}=\mathbf{N} \mathbf{U}
$$

where, $\mathbf{N}$ is the interpolation function of the finite element method.

According to the incremental theory in the elastoplasticity, 
the above three equations can be transformed into the following discretized forms:

$$
\{\mathbf{K}\}_{i}\{\Delta \mathbf{U}\}_{i}=\{\mathbf{P}\}_{i}
$$

where $\{\mathbf{K}\}_{i},\{\Delta \mathbf{U}\}_{i}$ and $\{\mathbf{P}\}_{i}$ are the stiffness matrix, the incremental displacement vector and the load vector in the $i$-th step, respectively.

$\{\mathbf{K}\}_{i}$ can be expressed as:

$$
\{\mathbf{K}\}_{i}=\sum_{e}\left(\int_{\Omega^{e}} \mathbf{N}^{T} \mathbf{L}^{T}\left\{\mathbf{D}^{e p}\right\}_{i} \mathbf{L} \mathbf{N} d \Omega\right)
$$

where, $\left\{\mathbf{D}^{e p}\right\}_{i}$ is the elastoplastic tensor in the $i$-th step.

$\{\mathbf{P}\}_{i}$ can be expressed as:

$\{\mathbf{P}\}_{i}=\sum\left(\int_{\Omega^{e}} \mathbf{N}^{T} \mathbf{L}^{T}\left\{\mathbf{D}^{e p}\right\}_{i}\left\{\Delta \varepsilon_{0}\right\}_{i} d \Omega+\int_{\Omega^{e}} \mathbf{N}^{T} \cdot\{\mathbf{F}\}_{i} d \Omega+\int_{\Gamma^{e}} \mathbf{N}^{T} \cdot\{\mathbf{T}\}_{i} d \Gamma\right)(\bar{\gamma}$ where $\left\{\Delta \varepsilon_{0}\right\}_{i},\{\mathbf{F}\}_{i}$ and $\{\mathbf{T}\}_{i}$ are the strain of initial tensor, the body force and the surface force in the $i$-th step, respectively.

$\left\{\Delta \varepsilon_{0}\right\}_{i}$ can be calculated by the temperature variation in the $i$-th step $\{\Delta T\}_{i}$ and the thermal expansion coefficient $\alpha$, as shown in Eq. (8):

$$
\left\{\Delta \varepsilon_{0}\right\}_{i}=\left\{\begin{array}{llllll}
1 & 1 & 1 & 0 & 0 & 0
\end{array}\right\}^{T} \alpha\{\Delta T\}_{i}
$$

In the mold surface element method, the reaction from the mold to the casting correlates to the displacement of the surface of the casting, as shown in Eq. (9).

$\{\mathbf{T}\}_{i}=-P_{m} \mathbf{n}=-\{\beta\}_{i} \delta \mathbf{n}=-\{\beta\}_{i}\left(\mathbf{n} \cdot\{d \mathbf{u}\}_{i}\right) \mathbf{n}=-\{\beta\}_{i} \mathbf{n} \otimes \mathbf{n} \cdot \mathbf{N}\{\Delta \mathbf{U}\}_{i}(\Phi$

where, $\mathbf{n}$ is the normal of the surface element, minus means the direction of the force is opposite to $\mathbf{n}, \otimes$ is the operation of tensor product, $\{d \mathbf{u}\}_{i}=\mathbf{N}\{\Delta \mathbf{U}\}_{i}$ is the incremental displacement of the surface elements in the $i$-th step, $\{\beta\}_{i}$ is the effective stiffness coefficient of the mold in the $i$-th step, which is expressed as:

$$
\{\beta\}_{i}=\left\{\begin{array}{l}
\beta_{m}, \text { when } \mathbf{n} \cdot\{\mathbf{u}\}_{i-1}>0 \\
0, \text { when } \mathbf{n} \cdot\{\mathbf{u}\}_{i-1}<0
\end{array}\right.
$$

where, $\beta_{m}$ is the stiffness coefficient of the mold, $\{\mathbf{u}\}_{i-1}$ is the accumulated displacement until the $(i-1)$ th step. It is expressed as:

$$
\{\mathbf{u}\}_{i-1} \approx\{d \mathbf{u}\}_{1}+\{d \mathbf{u}\}_{2}+\cdots+\{d \mathbf{u}\}_{i-1}=\sum_{1}^{i-1}\left(\mathbf{N}\{\Delta \mathbf{U}\}_{j}\right)
$$

The aforementioned equations constitute the models in the numerical simulation of casting thermal stress. By solving the linear system shown in Eq. (5), the incremental displacement in each step can be calculated. Then the total displacement, total strain and total stress can be calculated by using Eq. (12), Eq. (13) and Eq. (14), respectively.

$$
\begin{gathered}
\{u\}_{\text {total }} \approx \sum_{i}\left(\mathbf{N}\{\Delta \mathbf{U}\}_{i}\right) \\
\{\varepsilon\}_{\text {total }}=\sum_{i}\{\Delta \varepsilon\}_{i} \\
\{\sigma\}_{\text {total }}=\sum_{i}\left(\{\Delta \sigma\}_{i}\right)=\sum_{i}\left(\left\{\mathbf{D}^{e p}\right\}_{i}\{\Delta \varepsilon\}_{i}\right)
\end{gathered}
$$

\section{Test case for MSE method}

The stress lattice casting with multiple cores of different hardness was used to examine the applicability and correctness of the MSE method. Meanwhile, the results from PROCAST were used to validate the MSE method.

Figure 2 shows the geometric model of the stress lattice with cores of different hardness, among which the blue core is made of hard material (i.e. resin sand) and the red core is made of soft material (i.e. sodium silicate sand). Table 1 demonstrates the parameters in the stress lattice casting process.

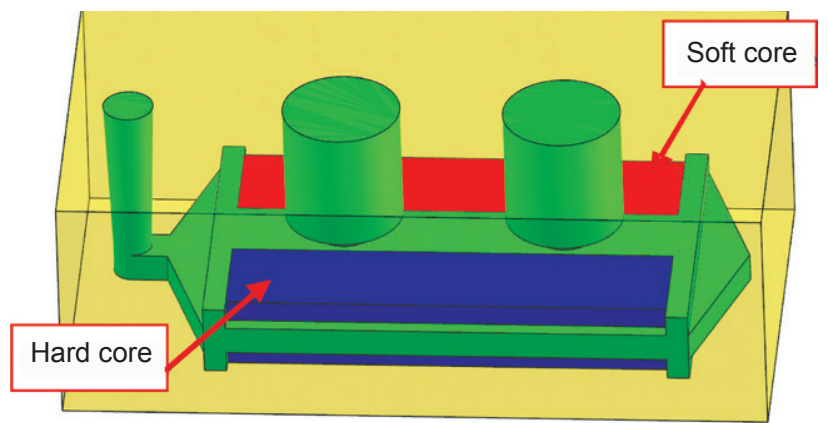

Fig. 2: Geometric model of stress lattice casting process with cores of different stiffness

Table 1: Parameters of stress lattice casting process

$\begin{array}{ccccc} & \text { Material } & \text { Elastic modulus at } & \text { Poisson ratio } & \text { Initial temperature }\left({ }^{\circ} \mathbf{C}\right) \\ \text { Casting } & \text { ZG25 } & 200 & 0.27 & 1,550 \\ \text { Hard core } & \text { Resin bonded sand } & 100 & 0.3 & 20 \\ \text { Soft core } & \text { Sodium silicate bonded sand } & 10 & 0.3 & 20 \\ \text { Mold } & \text { Resin bonded sand } & 100 & 0.3 & 20\end{array}$

Figure 3 shows the modulus distribution on the mold surface elements which accord with the parameters in the casting process.

Figure 4 demonstrates the final distribution of the stress and deformation of the casting. In Fig. 4, because of the obstacle of the hard core, the thin bar of the stress lattice close to the hard core suffers from a great stress concentration and greater deformation; therefore it has a greater possibility of cracking.
Contrarily, the effective stress on the thin bar close to the soft core is much smaller than that on the other side as the soft core does not restrict the shrinkage of the casting.

The casting simulation software PROCAST applies the contact element method to describe the interaction between the casting and the mold. Here, the computation for the same test case shown in Fig. 2 by both the MSE method and PROCAST 


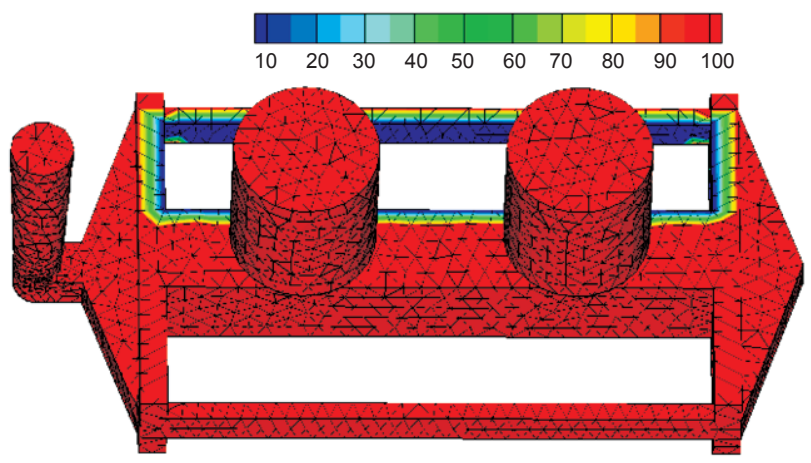

Fig. 3: Modulus distribution on mold surface elements

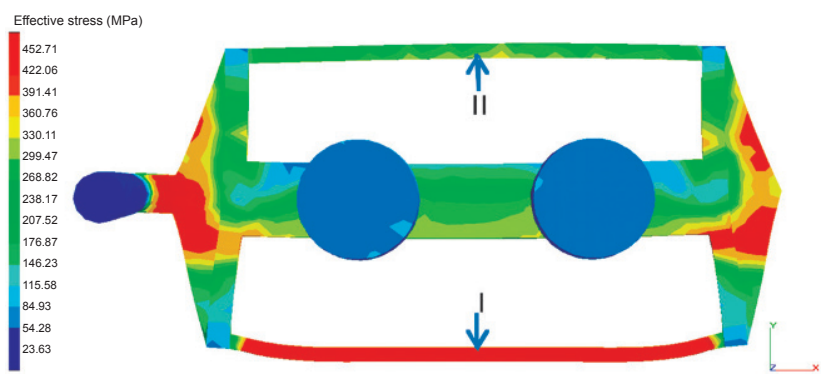

Fig. 4: Simulated stress distribution and deformation of stress lattice casting due to cores with different stiffness by MSE are compared under the same computation platform (CPU: Intel i7-4500; Memory: 8G). The result by PROCAST is shown in Fig. 5. Figures 4 and demonstrate similar distributions of thermal stress as well as the deformation. Table 2 shows the specific numerical comparisons. Here, the thin bar next to the hard core is marked as Bar I, and the other thin bar next to the soft core is marked as Bar II. Obviously, both the methods produced similar results. Because the MSE method uses surface elements $(19,488$ triangle elements) to describe the interaction between the casting and the mold, the computation consumption is dramatically reduced. The time consumed by the MSE method is merely $1 / 10$ of that of the contact element method.

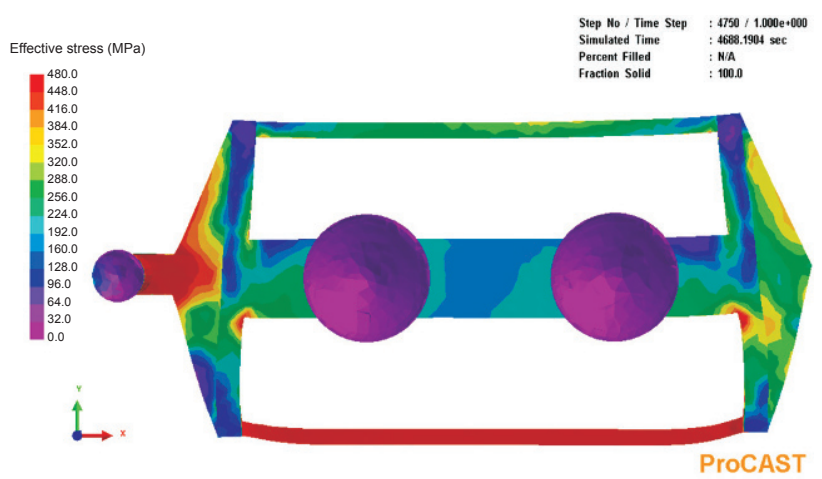

Fig. 5: Simulated stress distribution and deformation of stress lattice casting due to cores of different stiffness by PROCAST

Table 2: Results comparison between MSE and PROCAST methods

\begin{tabular}{|ccc|}
\hline & MSE method & Contact element method in PROCAST \\
\hline The largest equivalent stress on Bar I (MPa) & 452 & 487 \\
The largest equivalent stress on Bar II (MPa) & 331 & 362 \\
The largest equivalent plastic strain on Bar I (MPa) & $5.67 \mathrm{E}-2$ & $5.20 \mathrm{E}-2$ \\
The largest equivalent plastic strain on Bar II (MPa) & $2.23 \mathrm{E}-4$ & 61,059 tetrahedron elements \\
The number of elements in casting & 61,059 tetrahedron elements & 157,561 tetrahedron elements \\
The number of elements in mold and cores & 19,488 triangle elements & 573
\end{tabular}

This test case shows that the MSE method is capable of describing the complex boundary conditions between the casting and the mold. Meanwhile the precision of the MSE method is close to that of the contact element method.

\section{Verification of MSE method}

Two castings were simulated with the MSE method, and the results were compared with the real castings. The first one is a casting used on trains. As shown in Fig. 6, there is a depression at the center of one face of the casting. Figure 7 illustrates the numerical simulation results. In Fig. 7, the deformation of the casting has been magnified by 5 times so as to show the concavity more clearly.

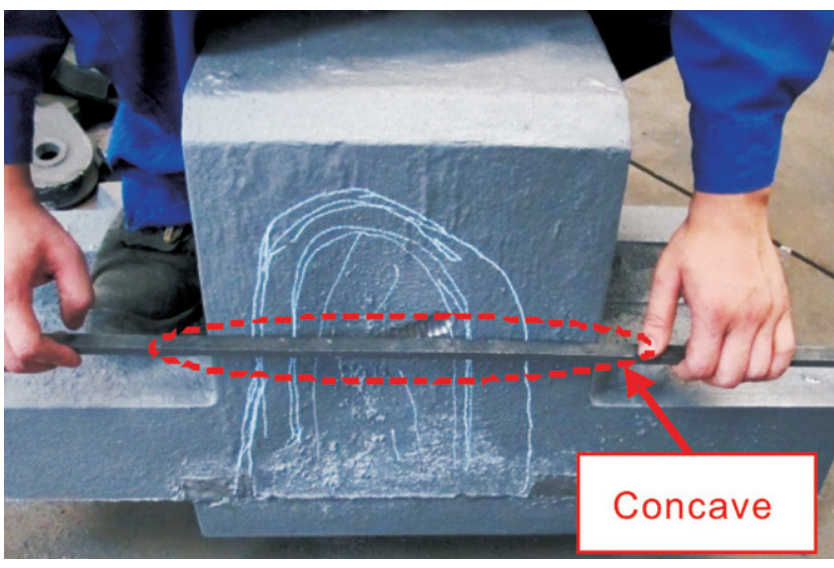

Fig. 6: Concave on surface of a product 


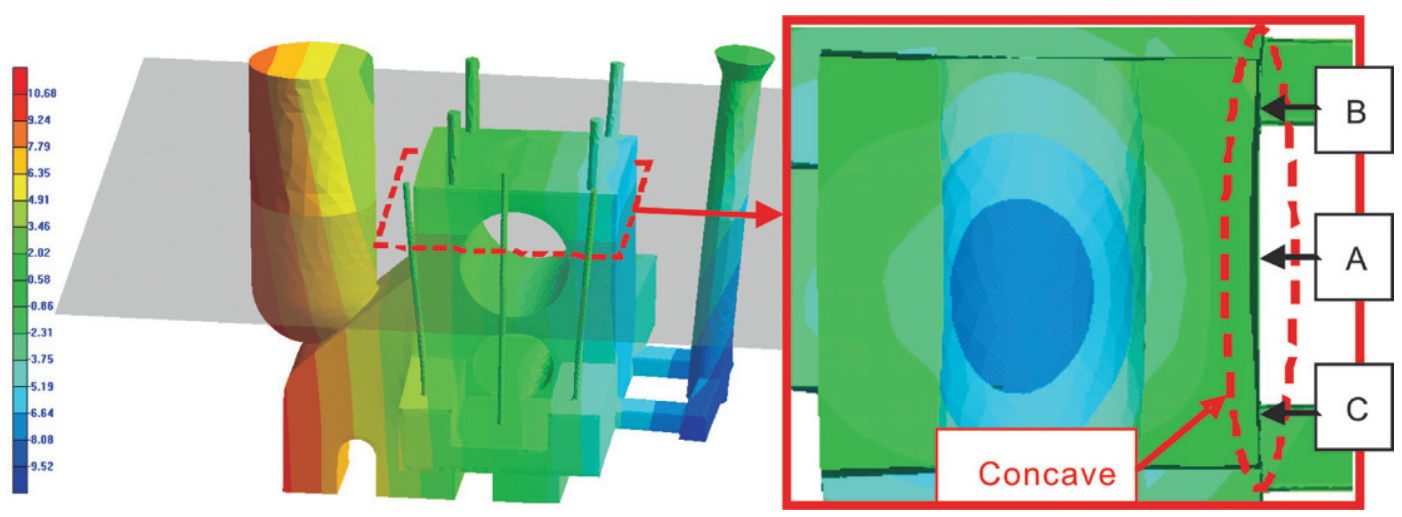

Fig. 7: Simulative concave on surface of a product

Three positions, marked as Point A, Point B and Point $\mathrm{C}$ are selected on the concavity. Point $\mathrm{A}$ is located at the center of the depressed face, while Point $\mathrm{B}$ and Point $\mathrm{C}$ are at the edges of the concavity. Figure 8 shows the evolution of the displacement along the $\mathrm{X}$ axis at three points on the concavity of the product. As the casting solidifies and shrinks, the displacement along the $\mathrm{X}$ axis at Points $\mathrm{A}, \mathrm{B}$ and $\mathrm{C}$ become smaller. Before $116 \mathrm{~s}$ during the solidification, the shrinkage at Point A is less than those at Points B and C. However, the shrinkage at Point A accelerates after $116 \mathrm{~s}$ and becomes larger than those of Points B and C. After solidification, the shrinkage at Point A is $1 \mathrm{~mm}$ more than those at Points B and C, resulting in the depression. This analysis by numerical simulation accords well with the results in the experiments.
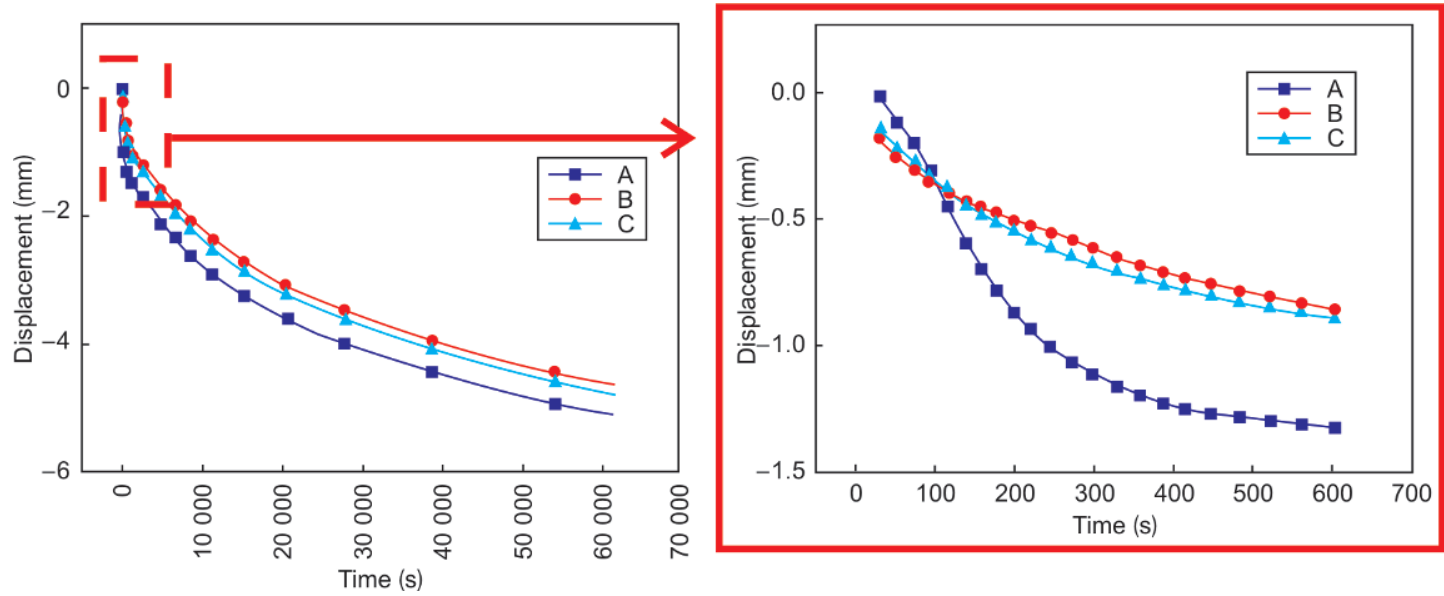

Fig. 8: Evolution of displacement along $X$ axis at three points on concavity of product

Table 3 shows the deformation results from the MSE method and the experiment. It is clear that the simulation result accords well with that of the experiment.

Table 3: Results comparison between MSE method and experiment (depth at point $A, \mathrm{~mm}$ )

\section{Simulated by MSE}

0.81
Figure 9 illustrates another geometric model of the product used in the train. This product features several planes. However, there are some concaves on the upper side of the real product, as shown in Fig. 10. The results of numerical simulation on the left of Fig. 10 successfully predicted the concave problem found in the reality.

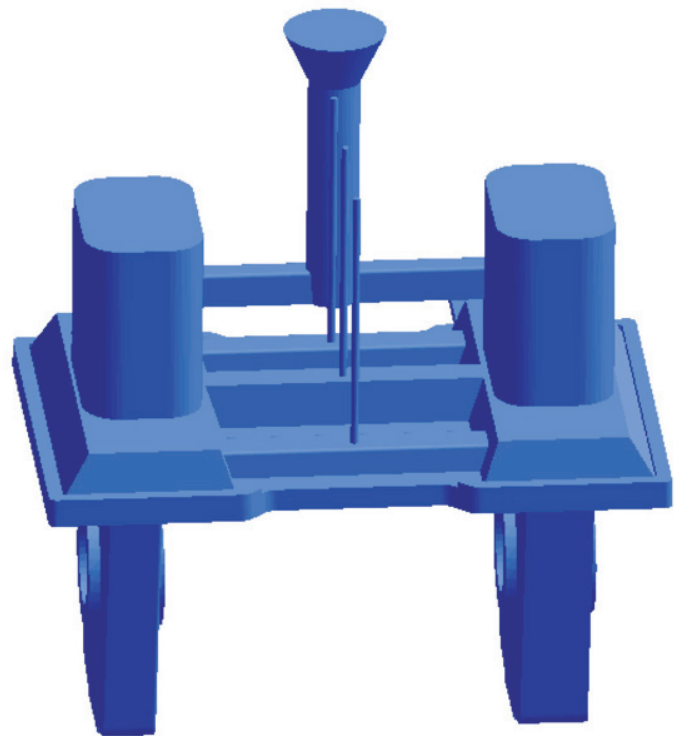

Fig. 9: Geometric model of a casting process 


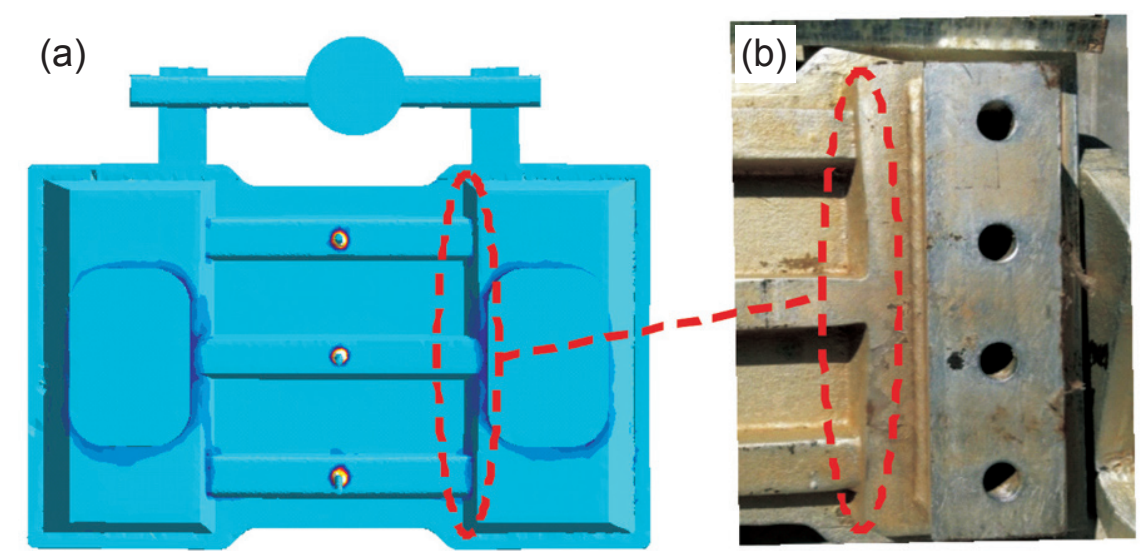

Fig. 10: Comparison of deformation in MSE simulation (a) and experiment (b)

\section{Conclusion}

This paper presents a new kind of approach called mold surface element (MSE) method, which deems the mold as being boundary elements around the casting. The method can describe the complex boundary condition of the mold efficiently and effectively, such as the casting process with mold parts of several different kinds of materials. As there is no calculation for the strain and the stress of the mold, the total computation can be obviously reduced, and the efficiency and convergence can be improved. All the restrictions of displacement are set automatically, assuring the non-singularity of the stiffness matrix. Two practical products are used to verify the applicability and accuracy of the MSE method. The results show that the MSE method successfully predicts the deformation of the casting found in the reality, providing guidance for process optimization.

\section{References}

[1] Jiang Ruisong, Zhang Dinghua, and Wang Wenhu. An integrated system for investment casting mould design of aeroengine turbine blade. China Foundry, 2008, 2(02): 114-118.

[2] Dou Yangqing, Bu Kun, Dou Yangliu, et al. Reversing design methodology of investment casting die profile based on ProCAST. China Foundry, 2010, 02(2): 132-137.

[3] Liao Dunming, Zhang Bin, Zhou Jianxin, et al. Using finite difference method to simulate casting thermal stress. China Foundry, 2011, 02(2): 177-181.

[4] Wang Yueping. Numerical simulation of thermal stress and deformation of a super-alloy blade investment casting. Master Dissertation, Harbin Institute of Technology, 2007.

[5] Cheng Jianguo, Kang Jinwu, Zhang Jiafeng, et al. Extraction of deformation of castings from simulated displacement results. Foundry Technology, 2008, 29(10): 1322-1326. (In Chinese)

[6] Li Hui, Shi Jiansong and Zhang Aiqin. Numerical simulation and deformation analysis of thermal stresses in stress frame.
Foundry, 2010, 59(1): 38-41. (In Chinese)

[7] Wang Peng. The Prediction and Control of Deformation of ZL205A Large Shell Cylindrical Castings. Master Dissertation, Harbin Institute of Technology, 2014.

[8] Cui Xinpeng, Zhang Cheng, Fan Shixi, et al. Numerical Simulation of Casting Deformation and Stress in the Ti-Alloy Parts with Framework Structure. Special Casting \& NonFerrous Alloys, 2015, 35(3): 258-259. (In Chinese)

[9] Wei Donghai, Li Kerui, Wu Xianlong, et al. Numerical Simulation and Experiment on Stress and Strain in Machine Tool Iron Castings. In: Proc. Symposium on Compacted Graphite Iron \& Machine Tool Iron Castings, 2012, Zibo, Shangdong.

[10] Kwak S Y, Lim C H, and Nam J H. Effects of displacement boundary conditions on thermal deformation in thermal stress problems. China Foundry, 2013, 3(3): 187-194.

[11] Yan J, Chen Liliang, Liao Dunming, et al. Study on Numerical Simulation of Casting Thermal Stresses Based on FDM. Acta Metallurgica Sinica (English Letters), 2004, 1(17): 7-10.

[12] Ren Fenghua. Study on Numerical Simulation Technology of Thermal Stress Field in Sand-Casting Process. Master Dissertation, North University of China, 2008.

[13] Zheng Xianshu, Yao Shan, and Jin Junze. Study of calculation model of mold resisting stress. Journal of Dalian University of Technology, 1996(06): 53-57.

[14] Song Yuhua, Yan Yongnian, Zhang Renji, et al. Boundary model between casting and mould and its influence on the dimensional accuracy analysis of precision casting. Journal of Engineering Manufacture, 2002, 8(216): 1123-1134.

[15] Xu Yan, Kang Jinwu, and Huang Tianyou. Application of Contact Element Method in Simulating Thermal Stress during Solidification of Casting. Foundry Technology, 2006, 27(5): 506-510. (In Chinese)

[16] Zavarise $G$ and Wriggers P. A superlinear convergent augmented Lagrangian procedure for contact problems. Engineering Computations. Int $\mathrm{J}$ for Computer-Aided Engineering, 1999, 16(1): 88-119.

[17] Barral P and Quintela P. A numerical algorithm for prediction of thermo-mechanical deformation during the casting of aluminium alloy ingots. Finite Elements in Analysis and Design, 2000, 34(2): 125-143.

This research is financially supported by the Program for New Century Excellent Talents in University (No. NCET-13-0229), the National Science \& Technology Key Projects of Numerical Control (No. 2012ZX04010-031, 2012ZX0412-011), Natural Science Foundation of Hubei Province, China (2011CDB279). 